\title{
DINI DERIVATIVES OF CONTINUOUS FUNCTIONS
}

\author{
ANTHONY P. MORSE
}

Let us agree that our space $R$ is the set of real finite numbers.

Although it is clearly possible for the upper right-hand derivative $D^{+} f$ of a function $f$ continuous on $R$ to $R$ to assume only the values \pm 1 , nevertheless something of interest can be said about intermediary values assumed by the Dini derivatives. For example an almost immediate consequence of Theorem 5 below is Theorem 1 .

1. ThEOREM. If $f$ is continuous on $R$ to $R,-\infty<\lambda<\infty$, the set

$$
\operatorname{Ex}\left[D^{+} f(x) \geqq \lambda_{1}\right.
$$

is dense, the set

$$
E x\left[D^{+} f(x)<\lambda\right]
$$

is nonvacuous, then the set

$$
\operatorname{Ex}\left[D^{+} f(x)=\lambda\right]
$$

has the power of the continuum.

Our reasoning is based on a category argument.

Let us agree now that $f^{*}(A)$ is the image under the function $f$ of the set $A$.

2. Lemma. If $C$ is a compact nondense set and $f$ is such a continuous function on $R$ to $R$ that

$$
f(x)>f(y)
$$

whenever $x$ and $y$ are such members of $C$ that $x<y$, then the set $f^{*}(C)$ is compact and nondense.

ProOF. Let $g$ be such a strictly decreasing continuous function on $R$ onto $R$ that

$$
g(x)=f(x) \quad \text { whenever } x \in C .
$$

Since $g$ is a continuous one-one mapping of $R$ onto itself, it follows that $g^{*}(C)$ is compact and nondense. In view of the fact that

$$
f^{*}(C)=g^{*}(C)
$$

the proof is complete.

Received by the editors June 11, 1953. 
3. THEOREM. If $f$ is continuous on $R$ to $R$, the set

$$
E x\left[D^{+} f(x) \geqq 0\right]
$$

is dense,

$$
N=E x\left[D^{+} f(x)<0\right]
$$

then the set

$$
N \cup f^{*}(N)
$$

is of the first category.

Proof. Let $\omega$ be the set of non-negative integers. For $n \in \omega$ let

$$
C_{n}=E x\left[f(x+h)-f(x) \leqq-2^{-n} \cdot h \text { whenever } 0 \leqq h \leqq 2^{-n}\right] .
$$

Since

$$
N \cup f^{*}(N)=\bigcup_{n \in \omega}\left[C_{n} \cup f^{*}\left(C_{n}\right)\right]
$$

the desired conclusion is a consequence of the statement.

Statement. If $n \in \omega$, then the set

$$
C_{n} \cup f^{*}\left(C_{n}\right)
$$

is of the first category.

Proof. Let $J$ be such a sequence of closed intervals that:

$$
\begin{gathered}
\operatorname{diam} J_{k}=2^{-n} \quad \text { whenever } k \in \omega ; \\
R=\bigcup_{k \in \omega} J_{k} .
\end{gathered}
$$

After noticing that $C_{n}$ is closed we check, for $k \in \omega$, that:

$$
C_{n} \cap J_{k}
$$

is compact and nondense; moreover

$$
f(x)>f(y)
$$

whenever $x$ and $y$ are such members of $C_{n} \cap J_{k}$ that $x<y$; consequently because of Lemma 2 the set

$$
f^{*}\left(C_{n} \cap J_{k}\right)
$$

is compact and nondense; and thus

$$
\left(C_{n} \cap J_{k}\right) \cup f^{*}\left(C_{n} \cap J_{k}\right)
$$

is compact and nondense.

Since 


$$
C_{n} \cup f^{*}\left(C_{n}\right)=\bigcup_{k \in \omega}\left\{\left(C_{n} \cap J_{k}\right) \cup f^{*}\left(C_{n} \cap J_{k}\right)\right\}
$$

we now see that

$$
C_{n} \cup f^{*}\left(C_{n}\right)
$$

is of the first category.

4. Theorem. If $f$ is continuous on $R$ to $R$, the set

$$
E x\left[D^{+} f(x) \geqq 0\right]
$$

is dense,

$$
\begin{gathered}
-\infty<a<b<\infty, \quad f(b)<f(a), \\
J=E x[a<x<b], \quad K=E y[f(b)<y<f(a)], \\
Z=J \cap E x\left[D^{+} f(x)=0\right],
\end{gathered}
$$

then the set

$$
K \sim f^{*}(Z)
$$

is of the first category.

Proof. For $y \in K$ let

$$
M(y)=\operatorname{Sup}\{J \cap E x[f(x)=y]\},
$$

and let

$$
\begin{gathered}
N=E x\left[D^{+} f(x)<0\right], \\
Z^{\prime}=E x\left[x=M(y) \text { for some } y \in K \sim f^{*}(N)\right] .
\end{gathered}
$$

Clearly

$$
K \sim f^{*}(N)=f^{*}\left(Z^{\prime}\right)
$$

and hence

$$
D^{+} f(x) \geqq 0 \quad \text { whenever } x \in Z^{\prime} .
$$

On the other hand if $x \in Z^{\prime}$ then $x \in J$ and

$$
f(t)<f(x) \quad \text { whenever } t \text { is such that } x<t<b .
$$

Consequently

$$
D^{+} f(x) \leqq 0 \quad \text { whenever } x \in Z^{\prime}
$$

and thus, because of (2),

$$
D^{+} f(x)=0 \quad \text { whenever } x \in Z^{\prime}
$$


We conclude with the help of (1) and Theorem 3 that

$$
\begin{aligned}
& Z^{\prime} \subset Z, \quad K \sim f^{*}(N)=f^{*}\left(Z^{\prime}\right) \subset f^{*}(Z), \\
& K \sim f^{*}(Z) \subset K \cap f^{*}(N) \subset f^{*}(N), \\
& K \sim f^{*}(Z) \text { is of the first category. }
\end{aligned}
$$

The proof is complete.

Since $K \cap B$ has the power of the continuum whenever $K$ is such a nonvacuous open set that $K \sim B$ is of the first category, it is now easy to verify Theorem 5 .

5. THEOREM. If $f$ is continuous on $R$ to $R$, the set

$$
E x\left[D^{+} f(x) \geqq 0\right]
$$

is dense, then either $f$ is strictly increasing or the set

$$
E x\left[D^{+} f(x)=0\right]
$$

has the power of the continuum.

As advertised, Theorem 1 follows easily by so defining $g$ on $R$ that

$$
g(x)=f(x)-\lambda \cdot x \quad \text { whenever } x \in R
$$

and then applying Theorem 5 to $g$.

6. Remarks.

.1 If $f$ is a continuous function on $R$ to $R$ whose difference quotients are unbounded from above and below in each nonvacuous open set, then the set

$$
J \cap E x\left[D^{+} f(x)=\lambda\right]
$$

has the power of the continuum whenever $J$ is a nonvacuous open set and $\lambda \in R$.

$.2 \mathrm{~W}$. H. Young showed in Messinger of Mathematics vol. 38 (1908-09) that the Weierstrass nondifferentiable continuous function $W$ has the property that the set

$$
E x\left[D^{+} W(x)<\infty\right]
$$

is of the first category. He raised the question: Is it countable? In view of .1 it clearly isn't.

Actually

$$
E x\left[D^{+} f(x)<\infty\right]
$$

is a first category set with the power of the continuum whenever $f$ is a continuous nondifferentiable function. 
.3 If we so define $F$ on $R$ that for $x \in R$

$$
F(x)=D^{+} W(x) \text { or } 0
$$

according as $\left|D^{+} W(x)\right| \leqq 1$ or $\left|D^{+} W(x)\right|>1$, then $F$ is a bounded Borelian function which is everywhere discontinuous and yet has the property that

$$
J \cap \operatorname{Ex}[F(x)=\lambda]
$$

has the power of the continuum whenever $J$ is a nonvacuous open set and $-1 \leqq \lambda \leqq 1$.

.4 If $f$ is continuous on $R$ to $R$ and

$$
E x\left[D^{+} f(x)<0\right]
$$

has power less than the continuum, then $f$ is a nondecreasing function.

.5 If $f$ is a continuous nondecreasing function on $R$ to $R$,

$$
\begin{array}{rlrl}
f^{\prime}(x) & =0 & \text { for almost all } x, \\
f(0) & <f(1), & \\
0 & \leqq \lambda \leqq \infty,
\end{array}
$$

then the set

$$
E x\left[D_{+} f(x)=\lambda\right]
$$

has the power of the continuum.

.6 However, the Cantor function $\psi$ has the property that the set

$$
\operatorname{Ex}\left[D^{+} \psi(x)=\lambda\right]
$$

is void whenever $0<\lambda<\infty$.

.7 Because of .6 it may be seen that Theorem 1 becomes false if " $D^{+}$" is replaced by " $D_{+}$".

UNiversity of CALIForNia, BERKELEY 